\title{
EndoUDA: A modality independent segmentation approach for endoscopy imaging
}

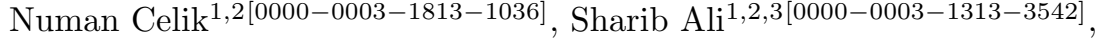 \\ Soumya Gupta ${ }^{1,2[0000-0001-5874-5273]}$, Barbara Braden2,3 [0000-0002-8534-6873], \\ Jens Rittscher ${ }^{1,2,3}[0000-0002-8528-8298]$ \\ 1 Department of Engineering Science, Institute of Biomedical Engineering, University \\ of Oxford, Oxford, UK \\ 2 Big Data Institute, University of Oxford, Li Ka Shing Centre for Health \\ Information and Discovery, Oxford, UK \\ 3 NIHR Oxford Biomedical Research Centre, Oxford, UK \\ 4 Translational Gastroenterology Unit, Experimental Medicine Div., John Radcliffe \\ Hospital, University of Oxford, Oxford, UK \\ \{numan.celik, sharib.ali, jens.rittscher\}@eng.ox.ac.uk
}

\begin{abstract}
Gastrointestinal (GI) cancer precursors require frequent monitoring for risk stratification of patients. Automated segmentation methods can help to assess risk areas more accurately, and assist in therapeutic procedures or even removal. In clinical practice, addition to the conventional white-light imaging (WLI), complimentary modalities such as narrow-band imaging (NBI) and fluorescence imaging are used. While, today most segmentation approaches are supervised and only concentrated on a single modality dataset, this work exploits to use a targetindependent unsupervised domain adaptation (UDA) technique that is capable to generalize to an unseen target modality. In this context, we propose a novel UDA-based segmentation method that couples the variational autoencoder and U-Net with a common EfficientNet-B4 backbone, and uses a joint loss for latent-space optimization for target samples. We show that our model can generalize to unseen target NBI (target) modality when trained using only WLI (source) modality. Our experiments on both upper and lower GI endoscopy data show the effectiveness of our approach compared to naive supervised approach and state-of-the-art UDA segmentation methods.
\end{abstract}

Keywords: Barrett's esophagus · Polyp · Endoscopy · Unsupervised domain adaptation $\cdot$ Variational autoencoder $\cdot$ Segmentation

\section{Introduction}

Most gastrointestinal (GI) cancers are preventable. In 2018, the five major types of GI cancers, which include those of the stomach, liver, oesophagus, and colorectum account for $26 \%$ of the global cancer incidence and $35 \%$ of all cancer deaths [2]. Endoscopy, a vital tool for screening and disease surveillance, is however heavily operator dependent and $12 \%$ of cancers are missed [11. Artificial 


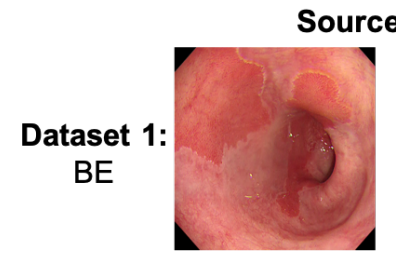

Input

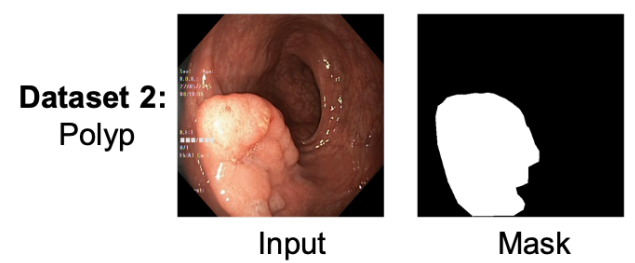

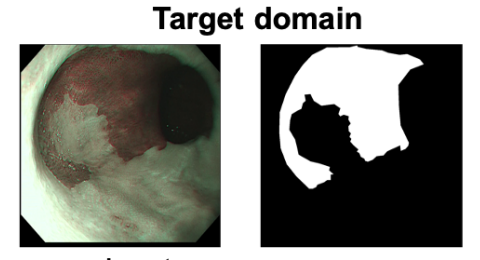

Mask

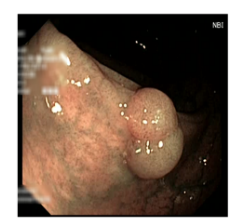

Input

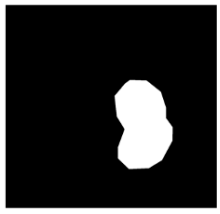

Mask

Fig. 1: Example images and masks of the two GI datasets for which source and target domains are separated based on their modality.

intelligence-based systems can play a vital role in improving diagnostic quality. However, it is critical that these tools integrate directly into the existing clinical workflow and generalize well to the previously unseen data.

Today, high-definition endoscopes provide a sufficient resolution to allow for a detailed visualisation of the mucosal surface. In addition to the conventional white light imaging (WLI) modality, autofluorescence imaging, and electronic chemoendoscopy techniques such as narrow-band imaging have been developed to improve the early detection of cancer and its precursors [15]. It is important that any computer assisted methods should work seamlessly in these different modalities and avoid the need for any modality-specific training. Developing such a modality agnostic approach for detection and segmentation of pre-cancerous changes in the esophagus, and polyps in the lower GI is the focus of this paper.

Convolutional Neural Network (CNN) based approaches [10!14 5, all well established in biomedical imaging, have been applied to segmentation tasks in GI endoscopy [1819. Wu et al. [19] proposed a dual neural network based on U-Net with ResNet backbone for automatic esophageal lesion segmentation. Guo et al. [8] utilized a FCN-based architecture with atrous kernels to segment polyps. However, all these supervised approaches are trained on WLI endoscopy and do not support working in different imaging modalities. To date, the problem of quantifying disease related changes in NBI, which can produce a very different appearance of the mucosal surface when compared to WLI (see Fig. 1) has not been addressed. Training methods for different imaging modalities independently is prohibitive. Firstly, extensive training datasets that cover a similar range of conditions would need to be curated to ensure a comparable performance. Secondly, manual annotations by human experts are required which is not only labour intensive but also expensive. To tackle these issues domain adap- 


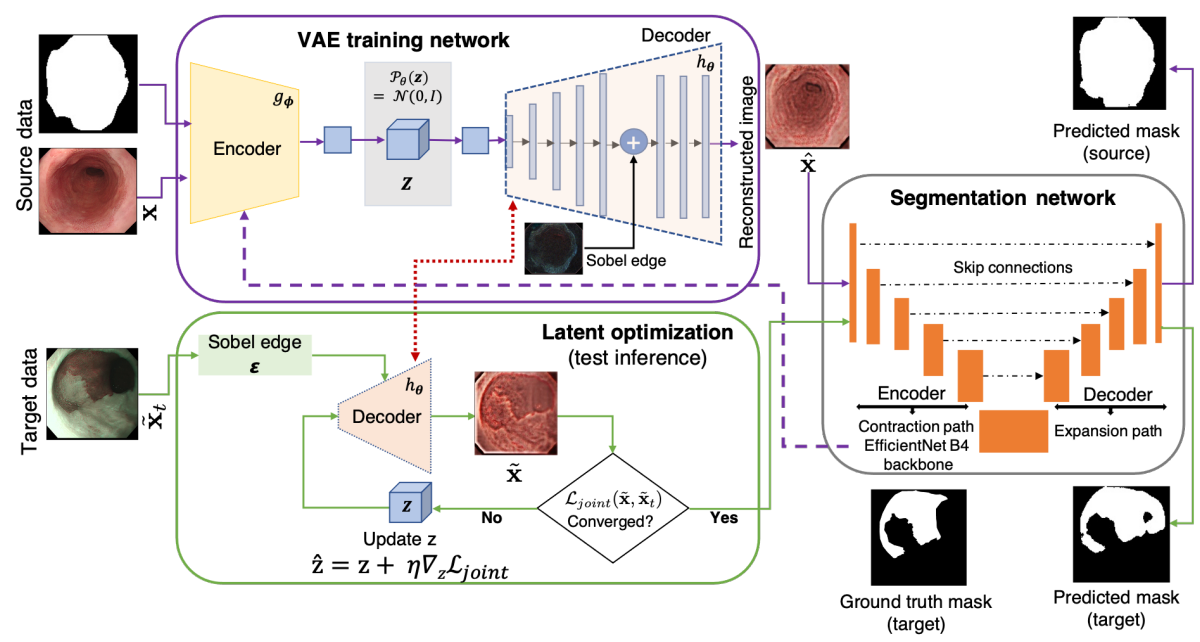

Fig. 2: Complete architecture of the proposed EndoUDA: a target-independent unsupervised domain adaptation.

tation methods have been used to adapt trained models to unseen target data for segmentation [17/79/13. Although these methods promise to generalise, so far there are only a few examples of applying such methods to medical data [79. Additionally, the available methods still under-perform and have not been adapted for extremely diverse modality cases such as that in the GI endoscopy.

To develop a modality-agnostic approach, we propose to use an unsupervised domain adaptation (UDA) technique that can be trained on available single WLI modality dataset and can be applied on an unseen NBI imagery. For such settings, where classical supervised models fail to generalize and perform poorly, we show that our proposed target-independent UDA technique, EndoUDA, has the potential to generalize and to provide improved segmentation on NBI modality for both BE and polyp datasets.

\section{Method}

Our proposed EndoUDA consists of components for training and test time inference shown in Figure 2. The training network, utilizes a shared EfficientNet-B4 encoder for our variational autoencoder (VAE) and a U-Net-based segmentation model. The VAE module allows to learn the source domain representations while U-Net module leverages the learned encoded features for semantic segmentation. For inference at test time, learnt latent space embeddings are further optimized to translate to the unseen modality dataset by continuously updating via a joint loss minimization scheme. Below, details of each of these modules are presented. 


\subsection{VAE training on source domain}

VAE is a latent space derived generative model that is based on variational inference distributions. Let $\mathcal{P}_{\theta}(\mathbf{z} \mid \mathbf{x})$ be the latent space distribution for sample $\mathbf{x}$ and $\mathcal{Q}_{\phi}(\mathbf{z} \mid \mathbf{x})$ be an identical tractable distribution so that it approximates the true posterior $\mathcal{P}_{\theta}(\mathbf{z} \mid \mathbf{x})$. Then, the variational lower bound term of the loss $\mathcal{L}(\theta, \phi)$ between probabilistic $h_{\phi}$ (encoder, outputs the parameters $\mu_{\mathbf{z}}$ and $\sigma_{\mathbf{z}}$ of a distribution) and $h_{\theta}$ (decoder) neural networks. In Eq. (1), approximated $\mathcal{Q}_{\phi}(\mathbf{z} \mid \mathbf{x})$ eliminates the non-negative KL-divergence term imposing a lower bound on the data log-likelihood only. This is maximized by the VAE via parameterizing $Q_{\phi}(\mathbf{z} \mid \mathbf{x})$ and $\mathcal{P}_{\theta}(\mathbf{x} \mid \mathbf{z})$ which is similar to the minimization of the reconstruction $\operatorname{loss} \mathcal{L}_{r}(\mathbf{x})$ in Eq. (2).

$$
\mathcal{L}(\theta, \phi)=\mathbb{E}_{Q_{\phi}}\left[\ln \left(\mathcal{P}_{\theta}(\mathbf{x} \mid \mathbf{z})\right)\right]-\mathbb{D}_{K L}\left[\mathcal{Q}_{\phi}(\mathbf{z} \mid \mathbf{x}) \| \mathcal{P}_{\theta}(\mathbf{z} \mid \mathbf{x})\right]
$$

Compared to the VAE framework in [13, we propose to couple the encoder layer of VAE as the segmentation network backbone. Based on our experiments, we have chosen an EfficientNet which computes the approximate posterior $\mathcal{Q}_{\phi}(\mathbf{z} \mid \mathbf{x})$. Unlike Pandey et al. 13, we remove the originally used perceptual loss module in the decoder layer used to compute the image likelihood of the observed data $\mathcal{P}_{\theta}(\mathbf{x} \mid \mathbf{z})$. The decoder network of the VAE contains 5 convolution layers followed by a LeakyRelu function (Leak $=0.2$ ) and a tanh activation function for the reconstructed output in the final convolution layer. A Sobel filter generated edge map is concatenated with the sixth layer of the decoder network of the VAE through a tanh nonlinear function (see Fig. 2). The reconstruction loss $\mathcal{L}_{r}(\mathbf{x})$ is minimized for the VAE training network given by:

$$
\mathcal{L}_{r}(\mathbf{x})=\mathcal{L}(\theta, \phi)=\frac{1}{N} \sum_{i=1}^{N}\left\|\mathbf{x}^{i}-h_{\theta}\left(h_{\phi}\left(\mathbf{x}^{i}\right)\right)\right\|^{2},
$$

where $\mathbf{x}$ is the source domain image, $N$ is total number of samples, $h_{\phi}$ and $h_{\theta}$ are encoder and decoder networks, respectively. Here, the reconstructed image is given by $\hat{\mathbf{x}}=h_{\theta}\left(h_{\phi}(\mathbf{x})\right)$. In addition, as shown in Fig. 2, the edges of the input images are extracted and concatenated into the decoder network in VAE through a skip connection to better generalize the source and target domains. Fig. 2 shows a schematic diagram of the complete VAE architecture used for training on the source domain.

\subsection{Segmentation module}

The segmentation network consists of a U-Net with an EfficientNet-B4 [16] backbone. The input from the reconstructed image obtained from the decoder of the VAE is fed to the already tuned weights of EfficientNet-B4. The segmentation network is adapted by a supervised loss on the source domain given by a classically used binary cross-entropy loss $\left(\mathcal{L}_{B C E}\right)$ and dice coefficient loss $\left(\mathcal{L}_{D S C}\right)$ [12]. $\mathcal{L}_{D S C}$ is represented as $\mathcal{L}_{D S C}=\left(2 \sum \mathbf{p g}\right) /\left(\sum \mathbf{p}^{2}+\sum \mathbf{g}^{2}\right)$, where $\mathbf{p}$ is the predicted probability map and $\mathbf{g}$ is the ground truth mask. 


\subsection{VAE latent search optimization via new joint Loss}

The trained VAE encoding aims to find the nearest point from the source domain, given a sample from the target domain, through an optimization process and updates of the latent space representation $\mathbf{z}$, as can be seen in Fig. 2 Once the decoder $h_{\theta}$ of the VAE is trained on the source distribution $\mathcal{P}_{s}(\mathbf{x})$, given an image $\tilde{\mathbf{x}}_{t}$ from the target distribution, the latent search algorithm finds the nearest point by optimizing latent vector over the iterative process. As shown in the Fig. 1, the WLI images are used in the source domain while the NBI are used in the target domain. For faster optimization convergence of the latent space encoding with improved generalization ability of the network, we propose to use a joint loss function that comprises of the normalized cross correlation $(\mathrm{NCC}) \mathcal{L}_{N C C}$ and the structural similarity index measure (SSIM) [21] loss $\mathcal{L}_{s s i m}$ between the image $\tilde{\mathbf{x}}_{t}$ from target domain and the reconstructed target image $\tilde{\mathbf{x}}$ from $h_{\theta}$. The loss function for computing NCC is defined as:

$$
\mathcal{L}_{N C C}\left(\tilde{\mathbf{x}}, \tilde{\mathbf{x}}_{t}\right)=\frac{1}{2 N} \sum\left(\frac{\tilde{\mathbf{x}}-\mu_{s}}{\sqrt{\sigma_{s}^{2}+\epsilon^{2}}}-\frac{\tilde{\mathbf{x}}_{t}-\mu_{t}}{\sqrt{\sigma_{t}^{2}+\epsilon^{2}}}\right)^{2},
$$

where $\tilde{\mathbf{x}}, \mu_{s}$ and $\sigma_{s}$ are reconstructed images from decoder network, mean and standard deviation of the latent encoded image, respectively. Similarly, $\tilde{\mathbf{x}}_{t}, \mu_{t}$ and $\sigma_{t}$ correspond to target image input, mean and standard deviation of the target image. The SSIM for a pair of images $\left(\tilde{\mathbf{x}}, \tilde{\mathbf{x}}_{t}\right)$ and loss function $\mathcal{L}_{s s i m}$ is defined as:

$$
\begin{aligned}
\operatorname{SSIM}\left(\tilde{\mathbf{x}}, \tilde{\mathbf{x}}_{t}\right) & =l\left(\tilde{\mathbf{x}}, \tilde{\mathbf{x}}_{t}\right)^{\alpha} * c\left(\tilde{\mathbf{x}}, \tilde{\mathbf{x}}_{t}\right)^{\beta} * s\left(\tilde{\mathbf{x}}, \tilde{\mathbf{x}}_{t}\right)^{\gamma}, \\
\mathcal{L}_{\operatorname{ssim}}\left(\tilde{\mathbf{x}}, \tilde{\mathbf{x}}_{t}\right) & =1-\operatorname{SSIM}\left(\tilde{\mathbf{x}}, \tilde{\mathbf{x}}_{t}\right),
\end{aligned}
$$

here $l, c$ and $s$ denote luminance, contrast and structure similarities of the given pair of images, respectively with the parameters $\alpha, \beta$ and $\gamma>0$. Also, the similarity loss function $\mathcal{L}_{\text {ssim }}$ is presented. The final joint loss function is given by:

$$
\mathcal{L}_{\text {joint }}=\lambda \mathcal{L}_{N C C}+(1-\lambda) \mathcal{L}_{\text {ssim }},
$$

where $\lambda=0.75$ is set empirically. After the convergence of $\mathcal{L}_{\text {joint }}$, the optimal latent space representation $\hat{\mathbf{z}}=\mathbf{z}+\eta \mathcal{L}_{\text {joint }}$ is used to generate the closest clone of source domain with a learning rate $\eta$ which is then used as an input to the segmentation network to predict the target mask.

\section{Experiments and results}

Implementation Details. For the training of our VAE network we used RMSProp with learning rate (lr) of 0.0001 while for the segmentation module Adam optimizer was used with $\beta_{1}=0.5, \beta_{2}=0.999$, and $l r=0.001$. The initial learning rate was reduced by a factor of 10 for every validation loss that did not improve in the last 3 epochs and a stopping criteria was set for no improvement in validation loss upto 10 epochs. We used 100 epochs for training our EndoUDA 
Table 1: Supervised Segmentation analysis: Benchmarking BE and polyp datasets on standard segmentation architectures on 80-20 train-test split.

\begin{tabular}{lcc|cc}
\hline \multirow{2}{*}{ Models } & \multicolumn{2}{c}{ BE } & \multicolumn{2}{c}{ Polyp } \\
\cline { 2 - 5 } & IoU & Dice & IoU & Dice \\
\hline U-Net[14] & 0.738 & 0.846 & 0.718 & 0.755 \\
UNet++[20] & 0.742 & 0.859 & 0.721 & 0.778 \\
ResUNet[6] & 0.789 & 0.908 & 0.734 & 0.794 \\
EfficientUNet [3] & $\mathbf{0 . 8 4 1}$ & $\mathbf{0 . 9 3 1}$ & $\mathbf{0 . 8 0 3}$ & $\mathbf{0 . 8 9 6}$ \\
EfficientUNet++ & 0.828 & 0.927 & 0.767 & 0.865 \\
DeepLabv3+ [5] & 0.802 & 0.893 & 0.789 & 0.869 \\
\hline
\end{tabular}

training module with batch size of 64 . For the test module of EndoUDA, we set learning rate of $\eta=0.001$ for optimization of the latent space representation and a fixed 40 iterations with batch size of 32 was set. The proposed method was implemented using the Tensorflow framework on a machine deployed with NVIDIA RTX 2080Ti graphics card with 12 GB GPU memory. All input images were resized to $128 \times 128$ pixels.

Datasets. We have used two gastrointestinal (GI) endoscopy datasets for analyzing two distinct endoscopically found precancerous anomalies, namely, Barrett's esophagus (BE) and polyps. To evaluate the efficacy of the proposed EndoUDA method, we have used clinically acquired white light imaging (WLI) and narrow-band imaging (NBI) modality data for BE and polyps. BE dataset consists of 1182 endoscopy images acquired from 68 unique patients of which 611 WLI images are used as source domain data (train set: $90 \%$, validation set: $10 \%$ ) and 571 NBI images as target domain data are used for testing. Similarly, for the polyp dataset, we used the publicly available Hyper-Kvasir dataset [4] for training which consists of 1000 images (train set: 80\%, validation set: 20\%). In addition, we used clinically acquired $42 \mathrm{NBI}$ images of polyps acquired from 20 patients at our local hospital and from collaborators.

\subsection{Benchmark comparison and ablation study}

Result of supervised segmentation of BE and polyps in our GI dataset are presented in Table 1. Frequently used supervised learning methods have been evaluated with respect to IoU and Dice to establish a baseline. It can be observed that the UNet with an EfficientNet B4 backbone 3] outperformed other baseline methods including DeepLabv3+ [5] by nearly $4 \%$ and $2 \%$ on both IoU and Dice for BE and polyps, respectively. Supplementary material Table 1-4 show that our choice of the joint loss function, the Sobel edge operator, the decoder architecture and the computational complexity yield in improved performance compared to alternatives that are often used. 
Table 2: Empirical results of EndoUDA along with SOTA UDA methods with mean $\mu$ and standard deviation $\sigma$ are provided. All comparisons are provided for source only trained model and tested on target data. Paired t-test between EndoUDA and each SOTA methods are shown in Supplementary Table 5.

\begin{tabular}{lllll|lllll}
\hline \multirow{2}{*}{ Models } & \multicolumn{4}{c|}{ BE $(\mu \pm \sigma)$} & \multicolumn{4}{c}{ Polyp $(\mu \pm \sigma)$} \\
\cline { 2 - 9 } & IoU & Dice & Precision & Recall & IoU & Dice & Precision Recall \\
\hline Naive U-Net [3] & 0.626 & 0.718 & 0.654 & 0.667 & 0.492 & 0.564 & 0.487 & 0.496 \\
& \pm 0.02 & \pm 0.015 & \pm 0.018 & \pm 0.021 & \pm 0.018 & \pm 0.012 & \pm 0.011 & \pm 0.011 \\
AdaptSegnet [17] & 0.658 & 0.749 & 0.721 & 0.698 & 0.519 & 0.577 & 0.601 & 0.583 \\
& \pm 0.019 & \pm 0.016 & \pm 0.012 & \pm 0.022 & \pm 0.017 & \pm 0.015 & \pm 0.016 & \pm 0.012 \\
ADVENT [18] & 0.667 & 0.768 & 0.739 & 0.706 & 0.524 & 0.591 & 0.662 & 0.638 \\
& \pm 0.012 & \pm 0.014 & \pm 0.015 & \pm 0.012 & \pm 0.011 & \pm 0.019 & \pm 0.021 & \pm 0.014 \\
CellSegUDA [9] & 0.673 & 0.771 & 0.733 & 0.691 & 0.533 & 0.629 & 0.688 & 0.641 \\
& \pm 0.02 & \pm 0.016 & \pm 0.018 & \pm 0.013 & \pm 0.012 & \pm 0.021 & \pm 0.018 & \pm 0.017 \\
GLSS [13] & 0.704 & 0.815 & $\mathbf{0 . 8 4 6}$ & 0.735 & 0.558 & 0.621 & 0.675 & 0.649 \\
& \pm 0.019 & \pm 0.022 & \pm 0.019 & \pm 0.02 & \pm 0.017 & \pm 0.016 & \pm 0.019 & \pm 0.012 \\
EndoUDA & $\mathbf{0 . 7 3 3}$ & $\mathbf{0 . 8 5 4}$ & 0.832 & $\mathbf{0 . 7 8 4}$ & $\mathbf{0 . 6 0 5}$ & $\mathbf{0 . 6 9 3}$ & $\mathbf{0 . 7 2 2}$ & $\mathbf{0 . 7 0 4}$ \\
& \pm 0.014 & \pm 0.021 & \pm 0.017 & \pm 0.019 & \pm 0.014 & \pm 0.02 & \pm 0.017 & \pm 0.018 \\
\hline
\end{tabular}
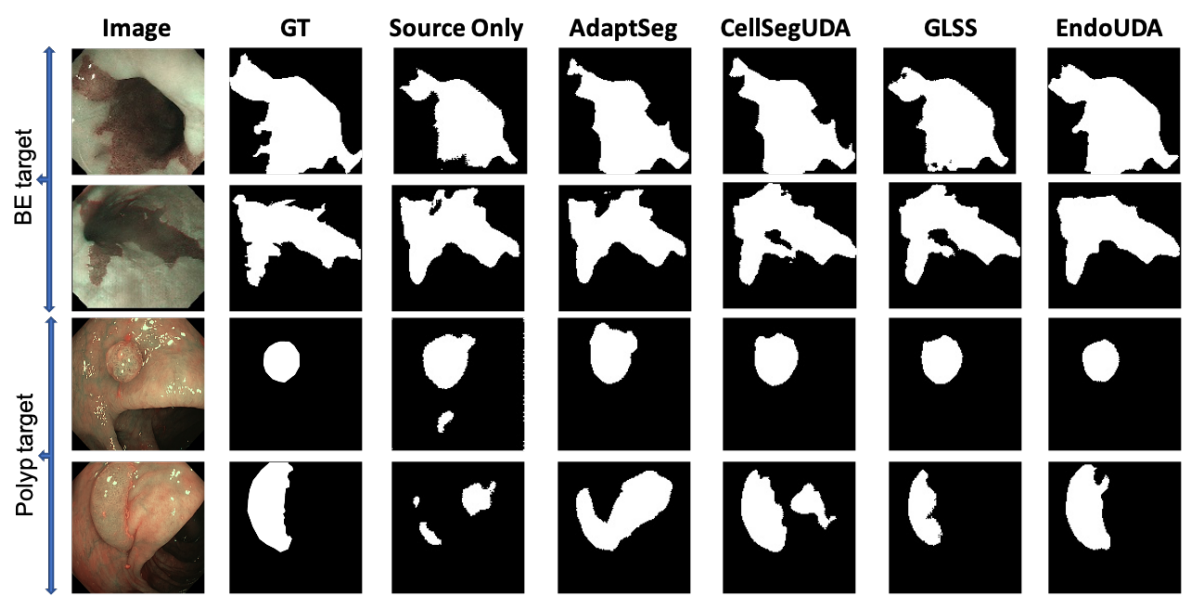

Fig. 3: Qualitative study: Baseline UDA experiments on BE and polyp images.

\subsection{Comparison with SOTA method}

We compared the proposed EndoUDA approach with four SOTA UDA methods: AdaptSegnet [17, ADVENT[18], CellSegUDA 9, GLSS[13, along with the naive supervised baseline model. The quantitative results are reported in Table 2 for four different metrics including intersection-over-union (IoU), Dice, precision and recall metrics. It can be observed that our proposed framework EndoUDA outperformed other SOTA UDA methods and naive U-Net method. An improve- 
Table 3: IoU and dice comparisons for out-of-distribution target samples (NBI) in the source (WLI) GI endoscopy datasets

\begin{tabular}{l|c|c|c|c}
\hline Method & $\mathrm{BE}_{\text {IoU }}$ & Polyp $_{\text {IoU }}$ & $\mathrm{BE}_{\text {dice }}$ & Polyp $_{\text {dice }}$ \\
\hline U-Net (source-only) & 0.626 & 0.492 & 0.718 & 0.564 \\
EndoUDA & 0.733 & 0.605 & 0.854 & 0.693 \\
U-Net (source 100\% + target 10\%) & 0.646 & 0.536 & 0.738 & 0.588 \\
EndoUDA (source 100\% + target 10\%) & 0.744 & 0.613 & 0.866 & 0.706 \\
U-Net (source 100\% + target 25\%) & 0.691 & 0.579 & 0.761 & 0.641 \\
EndoUDA (source 100\% + target 25\%) & 0.756 & 0.624 & 0.875 & 0.721 \\
U-Net (source 100\% + target 50\%) & 0.738 & 0.631 & 0.827 & 0.734 \\
EndoUDA (source 100\% + target 50\%) & 0.771 & 0.658 & 0.889 & 0.739 \\
U-Net (target-trained) & 0.841 & 0.802 & 0.931 & 0.896 \\
\hline
\end{tabular}

ment of nearly $4 \%$ on BE and $7 \%$ on polyp dataset for with respect to the Dice metric compared to the recent SOTA GLSS method. Similar improvements can be seen for other metrics for both GI datasets and also much higher improvements compared to other SOTA UDA methods. Table 5 in the supplementary material shows the statistical significance of the improvement provided by our method when compared to SOTA methods presented in Table 2 with p-values $<0.01$ for almost all metrics and SOTA approaches for both BE and polyp segmentation.

It can be seen in Fig. 3 that the predicted masks using proposed EndoUDA model for BE and polyp images are very close to the ground truth annotations compared to those predicted by other SOTA UDA methods.

\subsection{Effect of out-of-distribution data in supervised training}

We further investigated the performance of EndoUDA compared to the supervised baseline U-Net model (with EfficientNet backbone) by training the model with $10 \%, 25 \%$ and $50 \%$ of target data samples. It can be observed (see Table 3 that the EndoUDA performs consistently better than the baseline approach for a different proportion of target data inclusion in the training. For example, on $10 \%$ and $25 \%$ of target data mixed in the training sample, EndoUDA still provides nearly a $7-10 \%$ improvement on IoU and 8-13\% with respect to Dice scores over the baseline method.

\section{Conclusion}

We have presented a novel target-independent unsupervised domain adaptation VAE-based method that comprises of a shared encoder for both reconstruction and segmentation, and a joint loss function for improved unseen target domain generalization. We have validated our approach on two GI endoscopy datasets that often require additional modalities for screening and intervention. The proposed modality agnostic method can be used in clinical GI endoscopy without 
imposing any constraints on what imaging modality is being used. Our qualitative and quantitative results demonstrate the effectiveness and promise of the proposed method compared to both the naive baseline supervised model and state-of-the-art UDA segmentation methods.

Acknowledgement NC is supported by the Emerson Collective Cancer Research Fund, SG is funded by Boston Scientific, SA and JR are supported by National Institute for Health Research (NIHR) Oxford Biomedical Research Centre (BRC). The views expressed are those of the authors and not necessarily those of the NHS, the NIHR or the Department of Health. The authors declare no competing interests.

\section{References}

1. Ali, S., Dmitrieva, M., Ghatwary, N., Bano, S., et al.: Deep learning for detection and segmentation of artefact and disease instances in gastrointestinal endoscopy. Medical Image Analysis 70, 102002 (2021)

2. Arnold, M., Abnet, C.C., Neale, R.E., Vignat, J., Giovannucci, E.L., McGlynn, K.A., Bray, F.: Global burden of 5 major types of gastrointestinal cancer. Gastroenterology 159(1), 335-349 (2020)

3. Baheti, B., Innani, S., Gajre, S., Talbar, S.: Eff-UNet: A Novel Architecture for Semantic Segmentation in Unstructured Environment. In: IEEE Conference on Computer Vision and Pattern Recognition Workshops (CVPRW). pp. 1473-1481 (2020)

4. Borgli, H., Thambawita, V., Smedsrud, P.H., Hicks, S., et al.: HyperKvasir, a comprehensive multi-class image and video dataset for gastrointestinal endoscopy. Scientific Data 7(1), 1-14 (2020)

5. Chen, L.C., Zhu, Y., Papandreou, G., Schroff, F., Adam, H.: Encoder-Decoder with Atrous Separable Convolution for Semantic Image Segmentation. In: Proceedings of the European Conference on Computer Vision (ECCV). pp. 833-851 (2018)

6. Diakogiannis, F.I., Waldner, F., Caccetta, P., Wu, C.: ResUNet-a: a deep learning framework for semantic segmentation of remotely sensed data. ISPRS Journal of Photogrammetry and Remote Sensing 162, 94-114 (2020)

7. Dong, N., Kampffmeyer, M., Liang, X., Wang, Z., Dai, W., Xing, E.: Unsupervised Domain Adaptation for Automatic Estimation of Cardiothoracic Ratio. In: Medical Image Computing and Computer Assisted Intervention (MICCAI). pp. 544-552 (2018)

8. Guo, Y., Bernal, J., J Matuszewski, B.: Polyp Segmentation with Fully Convolutional Deep Neural Networks - Extended Evaluation Study. Journal of Imaging 6(7), 69 (2020)

9. Haq, M.M., Huang, J.: Adversarial Domain Adaptation for Cell Segmentation. In: Proceedings of the Third Conference on Medical Imaging with Deep Learning (MIDL). pp. 277-287 (2020)

10. Long, J., Shelhamer, E., Darrell, T.: Fully convolutional networks for semantic segmentation. In: 2015 IEEE Conference on Computer Vision and Pattern Recognition (CVPR). pp. 3431-3440 (2015)

11. Menon, S., Trudgill, N.: How commonly is upper gastrointestinal cancer missed at endoscopy? A meta-analysis. Endoscopy international open 2(2), E46 (2014) 
12. Milletari, F., Navab, N., Ahmadi, S.: V-Net: Fully Convolutional Neural Networks for Volumetric Medical Image Segmentation. In: International Conference on 3D Vision (3DV). pp. 565-571 (2016)

13. Pandey, P., Tyagi, A.K., Ambekar, S., Prathosh, A.P.: Unsupervised Domain Adaptation for Semantic Segmentation of NIR Images Through Generative Latent Search. In: Proceedings of the European Conference on Computer Vision (ECCV). pp. 413-429 (2020)

14. Ronneberger, O., Fischer, P., Brox, T.: U-Net: Convolutional Networks for Biomedical Image Segmentation. In: Medical Image Computing and Computer-Assisted Intervention (MICCAI). pp. 234-241 (2015)

15. Subramanian, V., Ragunath, K.: Advanced endoscopic imaging: a review of commercially available technologies. Clinical Gastroenterology and Hepatology 12(3), 368-376 (2014)

16. Tan, M., Le, Q.: EfficientNet: Rethinking Model Scaling for Convolutional Neural Networks. In: Proceedings of International Conference on Machine Learning (ICML). pp. 6105-6114 (2019)

17. Tsai, Y., Hung, W., Schulter, S., Sohn, K., Yang, M., Chandraker, M.: Learning to Adapt Structured Output Space for Semantic Segmentation. In: IEEE Conference on Computer Vision and Pattern Recognition (CVPR). pp. 7472-7481 (2018)

18. Vu, T.H., Jain, H., Bucher, M., Cord, M., Pérez, P.: Advent: Adversarial entropy minimization for domain adaptation in semantic segmentation. In: 2019 IEEE/CVF Conference on Computer Vision and Pattern Recognition (CVPR). pp. 2512-2521 (2019)

19. Wu, Z., Ge, R., Wen, M., Liu, G., et al.: ELNet: Automatic classification and segmentation for esophageal lesions using convolutional neural network. Medical Image Analysis 67, 101838 (2021)

20. Zhou, Z., Siddiquee, M.M.R., Tajbakhsh, N., Liang, J.: UNet++: A nested u-net architecture for medical image segmentation. In: Deep learning in medical image analysis and multimodal learning for clinical decision support, pp. 3-11 (2018)

21. Zhou Wang, Bovik, A.C., Sheikh, H.R., Simoncelli, E.P.: Image quality assessment: from error visibility to structural similarity. IEEE Transactions on Image Processing 13(4), 600-612 (2004) 